ENGINEERING

\section{Smartphones sniff gases}

A common technology that enables short-range communication in smartphones could be used to detect airborne chemicals.

Near-field communication chips are found in half a billion mobile devices worldwide. They communicate wirelessly with small external tags and are used in contactless payment systems, for instance. A team at the Massachusetts Institute of Technology in Cambridge, led by Timothy Swager, modified the circuitry in the external tags using nanomaterials that are sensitive to certain chemicals. When a particular gas is present, the tag short-circuits and the smartphone can no longer read the tag.

By scanning combinations of tags, each of which was sensitive to a different chemical, the team could distinguish between gases including ammonia, hydrogenperoxide vapour and water vapour - down to the level of parts per million.

Such a system could be used to detect explosives or pollution and has other applications, the authors say.

Proc. Natl Acad. Sci. USA

http://dx.doi.org/10.1073/

pnas.1415403111 (2014)

\section{MOLECULAR EVOLUTION}

\section{Ancient apes digested ethanol}

Human ancestors were able to metabolize ethanol 10 million years ago, around the time that they came down from the trees.

Matthew Carrigan at Santa Fe College in Gainesville, Florida, and his co-workers analysed the gene encoding the enzyme ADH4, which

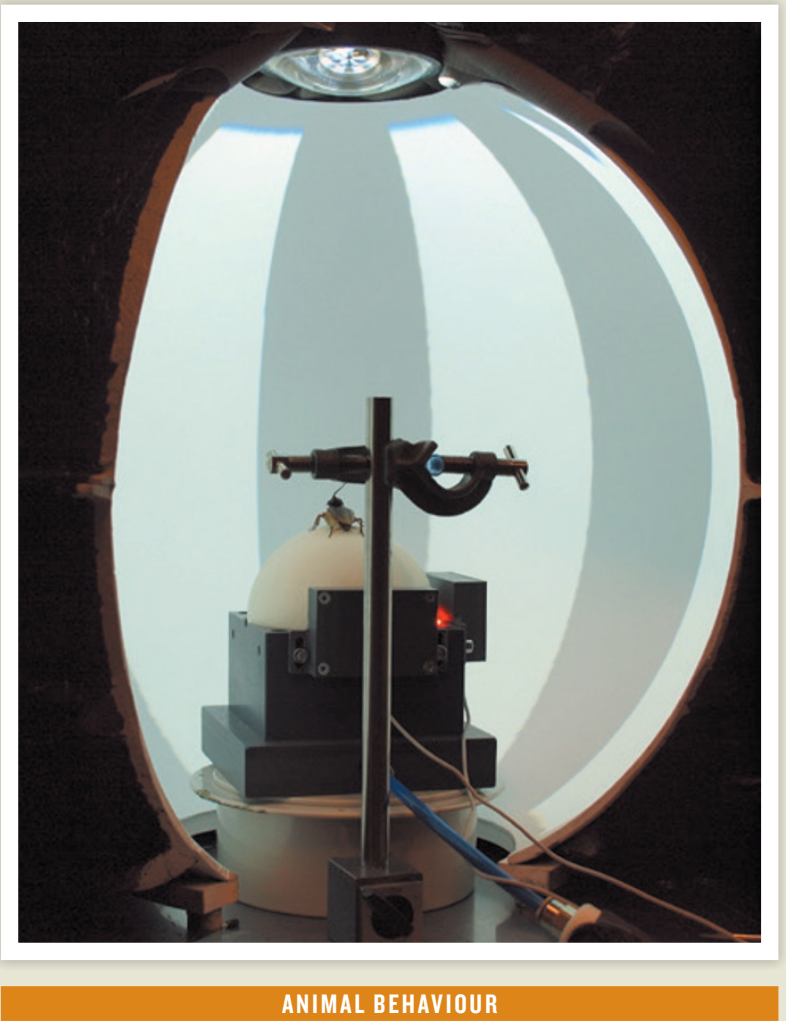

\title{
Cockroach night-vision
}

Cockroaches can see in near-darkness thanks to the many light-sensing cells in their eyes that pool a tiny number of light signals over space and time.

Matti Weckström and his colleagues at the University of Oulu, Finland, tested the behavioural responses of the American cockroach (Periplaneta americana) to varying levels of light, using a virtual-reality system that displayed moving patterns (pictured). By recording from individual light-sensitive eye cells, they found that each photoreceptor receives only one photon every 10 seconds when light levels are equivalent to a moonless night, during which the animals could still see. This pooling probably occurs over thousands of photoreceptors in the eye, say the authors.

Further study might improve night-vision devices, they add. J. Exp. Biol. 217, 4262-4268 (2014)

is made in the digestive tract to metabolize ethanol. They studied this gene from 28 mammals, including

17 primates, to trace its

70-million-year evolutionary history.

When they synthesized various ancestral forms of the enzyme, they found that $\mathrm{ADH} 4$ from ancestors of humans, chimpanzees and gorillas broke down ethanol much more efficiently than the enzyme from more ancient ancestors.

This change might have helped the hominids adapt to life on the forest floor, where there was probably more fermented fruit than in trees.

Proc. Natl Acad. Sci. USA http://doi.org/xkp (2014)

GLACIOLOGY

Antarctic ice loss accelerates

Glaciers flowing into

Antarctica's Amundsen Sea are some of the fastest melting on the continent - and in recent years have lost ice at an everquicker rate.

Different remote-sensing techniques have yielded slightly different estimates for the amount of ice melting from the Amundsen glaciers. Tyler Sutterley of the University of California, Irvine, and his colleagues compared and reconciled four ice-measuring methods. They found that between 2003 and 2009, the disappearance of Amundsen ice accelerated at a rate nearly three times faster than over the whole period between 1992 and 2013.

The findings boost confidence in the various ice-measuring methods and confirm just how quickly these glaciers are funnelling ice into the sea.

Geophys. Res. Lett. http://doi. $\mathrm{org} / \mathrm{xms}$ (2014)

\section{CRYOSPHERE}

\section{Melted Antarctic ice may not return}

The melting of ice around Antarctica as a result of global warming could be irreversible.

Jeff Ridley and Helene Hewitt of the UK Met Office's Hadley Centre in Exeter used a global climate model to examine how polar sea ice responds to changing climates. They found that Arctic sea ice melts and reforms in response 
to changing temperatures when carbon dioxide concentrations in the models are first increased and then gradually reduced to preindustrial levels. In Antarctica, however, sea ice returns at first, but had not recovered by the end of the simulation, even after a further 150 years of preindustrial $\mathrm{CO}_{2}$ levels.

This lack of ice recovery is a result of strong heat uptake by the Southern Ocean, which continues to warm parts of the seas around Antarctica long after global warming has been reversed, according to the authors.

Geophys. Res. Lett. http://doi. org/xh3 (2014)

\section{ANIMAL BEHAVIOUR}

\section{Electric eel zaps} neurons of its prey

The electric eel stuns its fish prey by emitting electrical pulses that control parts of the nervous system of its victim.

Kenneth Catania at

Vanderbilt University

in Nashville, Tennessee, studied the behaviour and electrical discharges of an eel (Electrophorus electricus; pictured) when it was presented with fish in an aquarium. He found that the

eel's shocks immobilize the fish

by activating nerves controlling the muscles, causing them to contract throughout the fish's body even when the fish's brain and spinal cord were destroyed. When the fish was hidden, the eel sent out two quick pulses, causing the fish to twitch, followed soon by a high-voltage zap and an attack.

The results show how the electric eel can remotely control its prey.

Science 346, 1231-1234 (2014)

$$
\text { IMMUNOLOGY }
$$

\section{How immune cells search and destroy}

To locate the source of an infection, immune cells called neutrophils take directions from local blood cells.

Neutrophils are the first responders to an infection, where they produce pathogenkilling compounds. To determine how they home in on infections and other injuries, a team led by Andrés Hidalgo at Spain's National Centre for Cardiovascular Research in Madrid imaged blood vessels in live mice that were showing an inflammatory response.

The authors discovered that neutrophils drifting in the bloodstream stuck to blood vessel walls and then sent out arm-like extensions. When these encountered blood cells called platelets - which are activated by injury to help to stop bleeding - the neutrophils began to migrate along the vessel wall and churn out toxic chemicals. Blocking communication between neutrophils and platelets lessened tissue damage from excessive inflammation in mouse models of sepsis, lung injury or stroke.

Science 346, 1234-1238 (2014)

\section{NEUROSCIENCE}

\section{Injury blunts brain waste disposal}

Fluid channels in the brain that help to remove waste could be impaired after traumatic injury, promoting cell death.

After injury, brain cells release a protein called tau, which accumulates as tangles and is associated with neurodegeneration and dementia. Jeffrey Iliff at the Oregon Health and Science

\section{SOCIAL SELECTION Romilumaticale}

\section{Funders drawn to alternative metrics}

In the digital age, a growing number of researchers and publishers are using more than just citation counts to track the impact of their articles. In an essay in PLoS Biology, three authors from a major UK research-funding agency argue that alternative metrics - or altmetrics, such as social-media mentions - can help funders to measure the full reach of the research that they support. Some researchers have already used these metrics in their favour. On his lab blog, Fernando Maestre, an ecologist at King Juan Carlos University in Madrid, explained how he included altmetrics in a successful grant proposal earlier this year. But not everyone is convinced that the new metrics are good for science. John Gilleard, a veterinary parasitologist at the University of Calgary in Canada, asked on Twitter: "Will an increased emphasis on \#altmetrics pressure researchers to 'over hype' their results?" PLoS Biol. 12, e1002003 (2014)

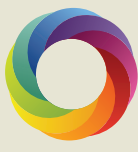

Based on data from altmetric.com. Altmetric is supported by Macmillan Science and Education, which owns Nature Publishing Group.

DNATURE.COM

For more on popular papers: go.nature.com/xep8gv

University, Portland, and

his colleagues

showed that tau

is cleared from young healthy mouse brains along the 'glymphatic pathway', channels that wash out waste from the brain.

The authors found that after traumatic injury, the pathway's performance decreased by about $60 \%$. It was reduced even further in injured mice in which a gene important for the pathway, aquaporin-4, had been knocked out. These mice developed tau tangles and performed less well in cognitive tests.

J. Neurosci. 34, 16180-16193 (2014)

\section{ANIMAL BEHAVIOUR}

\section{Some bats click wings to navigate}

Some bat species unable to use sonar to sense their environment can instead navigate using echoes from clicking their wings possibly an early, crude form of echolocation.

A team led by Arjan
Boonman and Yossi Yovel at Tel Aviv University in Israel studied three species of wild, non-echolocating Old World fruit bat (pictured is Cynopterus brachyotis). They found that individuals of two species emitted clicks more frequently in the dark than in the light, and could find and land on large objects, although they failed to detect small obstacles. When the researchers taped the bats' wings, the clicking stopped, but the exact clicking mechanism could not be determined.

The authors suggest that much can be learned about the evolution of echolocation from these fruit bats.

Curr. Biol. http://doi.org/xmr (2014)

\section{$\rightarrow$ NATURE.COM}

For the latest research published by Naturevisit:

www.nature.com/latestresearch 